\title{
Health related quality of life in children with congenital heart disease that undergo cardiac surgery during their first year of life
}

\author{
Melina J. Saavedra M.D..$^{a, b}$, Alfredo Eymann M.D. ${ }^{a}$, Lucía Pérez M.D. ${ }^{a}$, \\ Julio Busaniche M.D. ${ }^{a}$, Natalia Nápoli M.D. ${ }^{b}$, Pablo Marantz M.D. ${ }^{b}$ and Julián Llera M.D. ${ }^{a}$
}

\begin{abstract}
Introduction. Advances in diagnostic methods, surgical techniques and postoperative intensive care have significantly increased the survival rates for children with congenital heart disease. In this context, long term sequelae acquired greater relevance. Health-related quality of life (HRQOL) is a concept that helps to measure the impact of chronic diseases.

The purpose of the current study was to describe HRQOL in children with congenital heart disease that undergo cardiac surgery during their first year of life.

Methods. A cross-sectional observational study was conducted between August 2017 and December 2018 at a University General Hospital. PedsQL 4.0 Generic Core Scales were used.

Children with congenital heart disease between 2 and 4 years old who had cardiac surgery during their first year of life and healthy children were included.

Scores were compared with T-test or Wilcoxon according to the observed distribution. $p$ value $<0.05$ was considered significant.

Results. A total of 31 children with congenital heart disease ( $26 \%$ with a single ventricle) and 62 healthy children were enrolled. The first surgery was in the neonatal period in $61.3 \%$.

Our study showed no statistical differences $(p=0.10)$ between HRQOL Total Scale Score of children with congenital heart disease compared to healthy children. However, lower scores were observed with statistically significant differences in social $(\mathrm{p}=0.0092)$ and school $(\mathrm{p}=0.0001)$ scales. Conclusions. Our cohort of children diagnosed with congenital heart disease has a global quality of life comparable with healthy children except in social and school functioning scales. Key words: health related quality of life, congenital heart disease, cardiac surgery, chronic disease.

http:/ / dx.doi.org/10.5546/ aap.2020.eng.166
\end{abstract}

To cite: Saavedra MJ, Eymann A, Pérez L, Busaniche J, et al. Health related quality of life in children with congenital heart disease that undergo cardiac surgery during their first year of life. Arch Argent Pediatr 2020;118(3):166-172.

Funding: None.

Conflict of interest: None.

\section{INTRODUCTION}

Congenital heart diseases are malformations with an estimated incidence of 8 per 1000 live births. Symptomatic congenital heart diseases during the first year of life have an incidence of 2.5 to 3 per 1000 live births; they show the highest mortality rate and require early surgical correction. ${ }^{1}$

Early diagnosis of congenital heart disease, resulting from the development of diagnostic methods such as fetal ultrasound and echocardiography, allows birth in prepared neonatal intensive care units. $^{2-4}$ The survival of these children has increased also as a result of advances in surgical techniques and postoperative intensive care. ${ }^{2-4}$ In this context, the neurodevelopmental outcomes and long term sequelae acquire greater relevance in these children. In the literature most studies reported more incidence of neurological damage as well as delayed cognitive and motor development compare with healthy children. ${ }^{5-8}$

The term health-related quality of life (HRQOL) had emerged in the 1990s, and describes the influence of health and disease on quality of life. ${ }^{9}$ The World Health Organization (WHO) defines quality of life as individuals' perception of their position in life in the context of the culture and value systems in which they live and in relation to their goals, expectations, standards and concerns. ${ }^{10} \mathrm{HRQOL}$ is composed of subjective characteristics, because the term incorporates the individual's perception or, in the case of pediatrics, 
parents' appreciation about their children. With the purpose of measuring this marker objectively, questionnaires were developed that measure and provide empirical evidence to help in the decision-making process in health. ${ }^{11}$ However, according to published studies, the severity of the disease and patient's condition not necessarily is associated with their perception of quality of life. ${ }^{12}$

In Argentina, some questionnaires that achieve objective HRQOL were validated. ${ }^{13,14}$

PedsQL is a generic instrument that can be performed by healthy children and chronic patients or their parents. It allows to compare the HRQOL between healthy and sick children and also to contrast different groups of pathologies.

The presence of chronic disease, the requirement of cardiac surgery at an early age, the need for frequent medical visits and daily medication, could restrict a child's activities and therefore affect their quality of life.

To our knowledge, there are no publications in Argentina that exclusively address HRQOL in pediatric patients with congenital heart disease. Therefore, the aim of the study was to describe HRQOL in children from 2 to 4 years with congenital heart disease that underwent cardiac surgery during their first year of life.

\section{PATIENT POPULATION AND METHODS}

A cross-sectional study was conducted between August 2017 and December 2018 in Pediatric Cardiology and General Pediatrics division at a University General Hospital with pediatric cardiac surgery, pediatric intensive care unit and cardiac surgical recovery area.

The group of children with congenital heart disease consisted in patients operated during the first year of life at the hospital who at the time of the questionnaire were from 2 to 4 years old.

A group of healthy children consisted in patients from 2 to 4 years old who attended to the outpatient pediatrics clinic at the hospital were also included.

The exclusion criteria for both groups were genetic syndromes and other major congenital malformations, prematurity less than 30 weeks of gestational age, pre-existing chronic diseases such as chronic renal failure, liver failure, metabolic and rheumatological diseases, diagnosis of autism spectrum disorder and refusal to participate. In addition, in the group of children with congenital heart disease those who required heart transplantation, cardiac catheterization with the purpose of correcting congenital heart defects and those who had surgery in another institution were excluded. In the group of healthy children, those who required hospitalization for another reason during the last year were also excluded.

Children with congenital heart disease were identified by the registry of the Division of Pediatric Cardiac Surgery during the period between December 2012 and August 2015. They were selected for convenience as they met the selection criteria. The electronic medical record was reviewed by a pediatric cardiology fellow.

The group of healthy children was selected by a convenience sample with 2:1 ratio (2 healthy children per patient with congenital heart disease) in the waiting room of the outpatient pediatrics clinic at the hospital. Questions were asked to identify children who met the selection criteria.

Parents or tutors that were invited to participate were those who attended outpatient cardiology or pediatric clinics or were contacted by telephone calls. Oral informed consent was carried out with those who agreed to participate.

\section{Measure}

The PedsQL Generic Core Scales, for toddlers (ages 2-4), comprehend 21 items grouped into 4 scales (physical, emotional, social, school functioning) and summary scores (total scale score, physical health summary score and psychosocial health summary score) answered following a Likert response scale $(0=$ never a problem, 1 = almost never a problem, $2=$ sometimes a problem, $3=$ often a problem y $4=$ almost always a problem). The questionnaire is self-administered and was answered by one of the parents or tutors. The answers were converted to a scale of 0 to 100 being the highest scores correspond to better HRQOL.

The results in both groups were evaluated in order to describing the frequency that relevant problems (often a problem, almost always a problem) were reported in each scale.

In addition, the following data was collected: child's sex, age at the time of surgery, age at the time of the questionnaire, parents' maximum educational level, parents and siblings cohabitants, city of origin, sex of the parent or tutor that answer the questionnaire, congenital heart disease diagnosis, number of cardiac surgeries during the first year of life, surgical complications and use of cardiological medication at the time of the questionnaire.

The study was approved by the Research 
Protocol Ethics Committee of our institution (Protocol number IRB00010193).

\section{Sample size and statistical analysis}

We considered the validation of the Argentinean Spanish version of the PedsQL TM 4.0 Generic Core Scales in children and adolescents with chronic diseases, to estimate the sample size. ${ }^{13}$ The total scale score mean of the group of children with congenital heart disease from 2 to 4 years old was 74 (SD 13), and the mean of the group of healthy children was 82 (SD 12). We estimated a study with 30 children with congenital heart disease and 60 healthy children for getting a $95 \%$ confidence interval and a power of $80 \%$ for a two-tailed test with a 1:2 ratio.

Categorical variables were expressed in absolute number and frequency and continuous variables in mean and standard deviation or median and interquartile range according to the observed distribution.

For the statistical analysis, the comparison between healthy children and children with congenital heart disease was performed using Wilcoxon test given the nonparametric distribution of the data. Analysis was performed using Stata 13.

\section{RESULTS}

The questionnaire was conducted on 31 children with congenital heart disease and 62 healthy children who met the selection criteria (See flowchart in Figure 1).
The demographic characteristics of both groups are shown in Table 1. The children's median age was 3.3 years old (IQR 2.8-4.2); $76 \%$ of the questionnaires were answered by mothers; $54 \%$ of parents presented high educational level with university studies concluded; $93 \%$ of the parents were cohabitants and most of them had one child $(51 \%)$ or two ( $42 \%) ; 95 \%$ of the group of healthy children attended kindergarten, while $80 \%$ of children with heart disease did it.

Children with heart disease were male in $61 \%$ of the cases; $51 \%$ came from Buenos Aires City and Buenos Aires`suburbs, the rest of them proceed from towns further away. Congenital heart defect diagnoses are shown in Table 2; $26 \%$ had single ventricle physiology of which $50 \%$ were in Fontan stage at the time of the questionnaire. The mean of outpatient cardiac follow-up was 41.4 months and $61.3 \%$ of patients were on cardiovascular medication. First surgery was performed in the neonatal period in $61.3 \%$. Regarding the number of surgeries at the time of the questionnaire, $51.3 \%$ presented one and $29 \%$ required three surgeries. The percentage of patients with postoperative complications was variable depending on the number of surgery. Nine patients $(29 \%)$ had complications in more than one of them.

Our study showed no statistical differences $(p=0.10)$ between HRQOL Total Scale Score of children with congenital heart disease compared to healthy children. However, lower scores were observed with statistically significant differences

FIGURE 1. Flow diagram of patients with congenital heart disease who had surgery

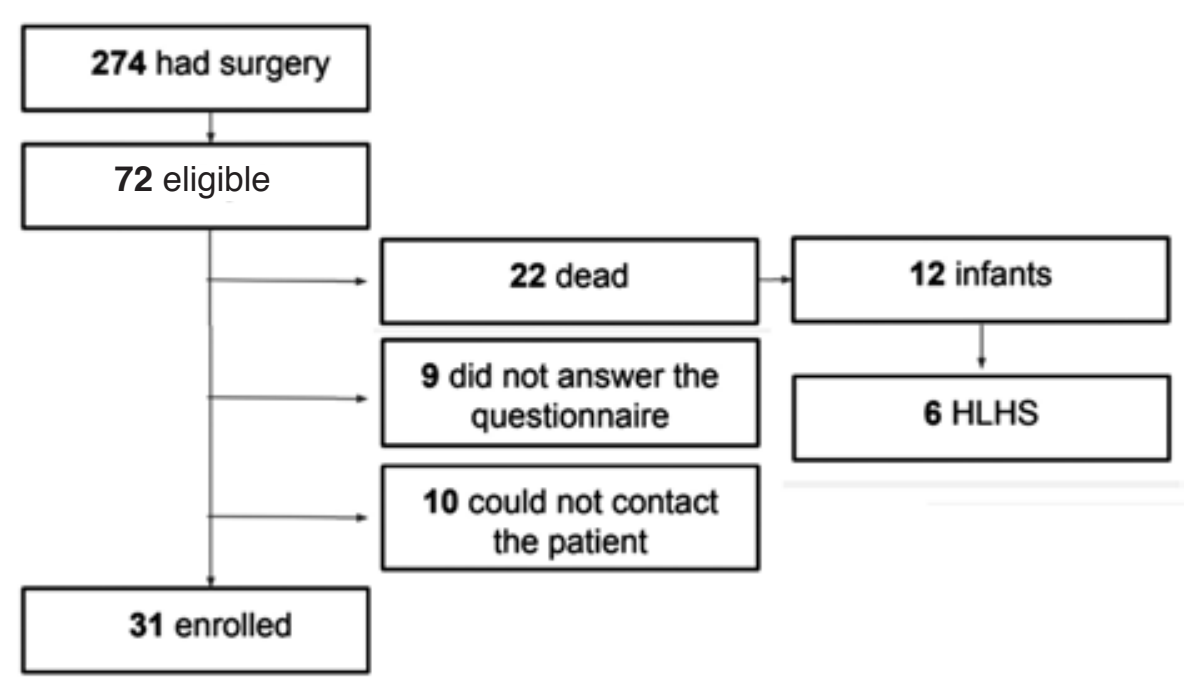

HLHS: hypoplastic left heart syndrome. 
in social $(\mathrm{p}=0.0092)$ and school $(\mathrm{p}=0.0001)$ scales (Table 3).

PedsQL responses show that $29 \%$ of parents of children with congenital heart disease reported relevant problems (often or almost always a problem) in at least one scale (Physical Functioning 6.4\%, Emotional Functioning $19.3 \%$, Social Functioning $9.6 \%$ and School Functioning $6.4 \%$ ). Otherwise, $17.7 \%$ of parents of healthy children reported these results (Physical Functioning 9.6\%, Emotional Functioning 9.6\%, Social Functioning $1.6 \%$, School Functioning $0 \%$ ).

\section{DISCUSSION}

Based on parents`reports, our study showed no statistical differences $(\mathrm{p}=0.10)$ between HRQOL Total Scale Score of the children from 2 to 4 years old with congenital heart disease operated during the first year of life compared to healthy children. Similar results were obtained in physical $(\mathrm{p}=0.88)$, psychosocial (0.43) and emotional (0.66) functioning scales. Lower scores were observed with statistically significant differences in social $(\mathrm{p}=0.0092)$ and school $(\mathrm{p}=0.0001)$ scales.
The aim of the current study was to describe HRQOL in children from 2 to 4 years with congenital heart disease that undergo cardiac surgery during their first year of life by using a validated tool, the PedsQL 4.0 Generic Core Scales.

HRQOL is a fundamental marker that measures the impact of chronic diseases. ${ }^{9}$ Studies about this topic are emerging worldwide. The results have been variable; several studies have reported lower HRQOL scores in children with congenital heart disease compared with other chronic diseases and healthy children..$^{5-8}$

Otherwise, some studies were found no significant differences between HRQOL in children with congenital heart disease and healthy children. ${ }^{15-19}$ Most of them involve patients who were diagnosed with transposition of the great vessels. Brosig et al., ${ }^{19}$ published a cohort similar to ours including 26 children who were diagnosed with hypoplastic left heart syndrome (HLHS) and transposition of great arteries (TGA) and used the PedsQL questionnaire. They have not found significant differences in Physical, Emotional and Social Scales. They have not reported the

TABLE 1. Demographic characteristics of healthy children and with congenital heart disease

\begin{tabular}{|c|c|c|c|}
\hline & Healthy children $(n=62)$ & Children with congenital heart disease $(n=31)$ & $p$ value \\
\hline \multicolumn{4}{|l|}{ Gender, n $(\%)$} \\
\hline Male & $32(51.6)$ & $19(61.3)$ & 0.37 \\
\hline Female & $30(48.4)$ & $12(38.7)$ & \\
\hline Age, median (IQR) & $3(2.58-4)$ & $3.83(3-4.41)$ & 0.04 \\
\hline \multicolumn{4}{|l|}{ Locality, n (\%) } \\
\hline Autonomus City of Buenos Aires & $56(90.3)$ & $6(19.4)$ & $<0.001$ \\
\hline Buenos Aires' suburbs & $6(9.6)$ & $10(32.3)$ & \\
\hline Less than $400 \mathrm{~km}$ & - & $3(9.7)$ & \\
\hline Between 400 and $1000 \mathrm{~km}$ & - & $6(19.4)$ & \\
\hline More than $1000 \mathrm{~km}$ & - & $6(19.4)$ & \\
\hline \multicolumn{4}{|l|}{$\begin{array}{l}\text { Gender of the parent who answer } \\
\text { the questionnaire, } \mathrm{n}(\%)\end{array}$} \\
\hline Male & $10(16.1)$ & $12(38.7)$ & 0.016 \\
\hline Female & $52(83.9)$ & $19(61.3)$ & \\
\hline \multicolumn{4}{|c|}{ Maximum educational level achieved, $\mathrm{n}(\%)$} \\
\hline Universitary & $37(59.7)$ & $14(45.5)$ & 0.6 \\
\hline Tertiary & $12(19.4)$ & $9(29)$ & \\
\hline High school & $12(19.4)$ & $8(25.8)$ & \\
\hline Primary school & $1(1.6)$ & $0(0)$ & \\
\hline Cohabit, n (\%) & $60(96.8)$ & $27(87.1)$ & 0.07 \\
\hline \multicolumn{4}{|l|}{ Siblings who cohabit, $\mathrm{n}(\%)$} \\
\hline 0 & $26(41.9)$ & $11(35.5)$ & 0.43 \\
\hline 1 & $26(41.9)$ & $13(41.9)$ & \\
\hline 2 & $7(11.3)$ & $5(16.1)$ & \\
\hline 3 or more & $3(4.8)$ & $2(6.4)$ & \\
\hline Attending to kindergarten, $\mathrm{n}(\%)$ & $59(95.1)$ & $25(80.6)$ & 0.02 \\
\hline
\end{tabular}


TABLE 2. Characteristics of children with congenital heart defects. $\mathrm{N}=31$

\begin{tabular}{lc}
\hline Diagnostic & $\mathrm{n}(\%)$ \\
\hline Ventricular septal defect (VSD) & $3(9.6)$ \\
Coarctation of the aorta & $2(6.45)$ \\
VSD + Coarctation of the aorta & $2(6.45)$ \\
Critical aortic stenosis & $1(3.2)$ \\
Shone complex & $2(6.45)$ \\
Atrioventricular septal defect & $1(3.2)$ \\
d-Transposition of the great arteries (d-TGA) & $2(6.45)$ \\
TGA + VSD & $2(6.45)$ \\
Truncus arteriosus & $1(3.2)$ \\
Tetralogy of Fallot (TOF) & $2(6.45)$ \\
Double outlet right ventricle (DORV) & $3(9.6)$ \\
Pulmonary atresia with VSD & $2(6.45)$ \\
Single ventricle* & $8(26)$ \\
Cardiaovascular medication, $\mathrm{n}(\%)$ & $19(61,3)$ \\
Age at first surgery (in months), $\mathrm{n}(\%)$ & \\
0 a 1 & $19(61.3)$ \\
1 a 3 & $6(19.3)$ \\
4 a 6 & $5(16)$ \\
7 a 9 & $1(3.3)$ \\
9 a 12 & - \\
Number of surgeries, $\mathrm{n}(\%)$ & \\
1 & \\
2 & \\
3 & \\
Global days with mechanical ventilation, $\mathrm{n}(\%)$ & \\
1 a 5 days & $16(51.5)$ \\
5 a 10 days & $7(22.5)$ \\
First surgery (n $=31)$ & $4(13)$ \\
Third surgery (n $=8)$ & $4(13)$ \\
\hline
\end{tabular}

${ }^{*}$ HLHS; tricuspid atresia type 1B; double inlet single ventricle + tricuspid atresia + pulmonary atresia; double inlet single ventricle + TGA + pulmonary stenosis; pulmonary atresia and intact ventricular septum. ** DORV Fallot type; DORV + coarctation of the aorta; DORV D + TGA + VSD.
School Score as much as the Total Scale Score, because most of the children had not attended kindergarten yet.

Our results are probably due to multiple factors. We obtained similar scores in Physical and Emotional Scales in both groups and it could be due to family's influence on the development of them. Otherwise, we reported significantly lower scores in Social and School Scales and it could be a consequence of children's interaction with society outside of the family circle. Schooling is other factor that could influence these results; the number of children diagnosed with congenital heart disease who attended kindergarten were lower $(p=0.02)$ and school absenteeism due to illness or scheduled medical checkups was much more frequent in that group compared with healthy children. Our group diagnosed with congenital heart disease lived $80 \%$ outside Autonomus City of Buenos Aires (CABA) $(p<0.001)$ and absenteeism increased if the family lives away from the hospital.

In some cohorts it was observed that the socioeconomic environment had an influence on HRQOL and mothers reported questionnaires' lower scores compared with fathers. ${ }^{20-24}$ In our study, there was a higher rate of fathers of children with congenital heart disease who answered the questionnaire compared with parents of healthy children $(p=0.016)$. Both groups had a high educational level $(\mathrm{p}=0.6)$ and were mostly cohabitants $(\mathrm{p}=0.07)$.

Studies in Argentina have described HRQOL in children with different chronic diseases such as asthma, celiac disease, liver and bone marrow transplantation, COPD, HIV, cancer, terminal chronic renal failure and congenital heart disease. Most of these children showed significantly lower scores in all scales compared with healthy children. ${ }^{13,25}$ These studies were mostly conducted in public hospitals. Scores in

TABLE 3. HRQOL in children with congenital heart defects and healthy children

\begin{tabular}{|c|c|c|c|}
\hline & Healthy children $(n=62)$ & Children with congenital heart defects $(n=31)$ & $p$ value \\
\hline Total & $92.5(87.5-96.25)$ & $89.7(75.8-95.4)$ & 0.10 \\
\hline Physical & $96.87(90.62-100)$ & $93.75(87.5-100)$ & 0.88 \\
\hline Psychosocial & $90(85-95)$ & $87.5(80-95)$ & 0.43 \\
\hline Emocional & $82.5(75-90)$ & $85(75-100)$ & 0.66 \\
\hline Social & $100(90-100)$ & $90(85-100)$ & 0.0092 \\
\hline School & $100(91.66-100)$ & $83.3(66.6-91.6)$ & 0.0001 \\
\hline
\end{tabular}

Values expressed in median and interquartile range. 
our study were higher compared with scores from the validation of the Argentinean Spanish version of the PedsQL. The mean score of our population with congenital heart disease was 86.32 versus 74.61 of the mencioned study and the mean score of healthy children was 91.42 versus $82.19 .{ }^{13}$ It is likely that our results are different given the fact that our study was conducted in a private general hospital of the community with population who had greater socioeconomic level and included a population from 2 to 4 years old.

Study limitations include the small number of patients and the fact that was conducted within a single center. Besides, the population compared belonged to different parts of the country and had differences in school attendance. We have used an instrument that measures HRQOL based on the opinion of the children's parents. It has been shown in some studies that this may differ from the reality that children perceive. ${ }^{26}$ Otherwise, there could be selection bias considering that parents who chose not to respond the questionnaire could have been influenced by a negative perception of the quality of life of their children.

HRQOL is an emerging medical indicator. Given the increase in the survival of patients with congenital heart disease, the interest to be able to estimate HRQOL begins to be part of the long-term plan of care of this population. Our study highlights the significance of HRQOL evaluation in chronic patients' follow-up. However, multicenter studies with a larger number of patients are needed in order to confirm these results.

\section{CONCLUSIONS}

Our cohort of children diagnosed with congenital heart disease has a global quality of life comparable with healthy children except in social and school functioning scales.

\section{Acknowledgements}

To the medical doctors Clara Ceriani and Julia Saulino for their collaboration.

\section{REFERENCES}

1. Hoffman JI, Kaplan S. The incidence of congenital heart disease. J Am Coll Cardiol. 2002; 39(12):1890-900.

2. Chock V, Lee HC. Neurodevelopmental outcomes for infants born with congenital heart disease. Neoreviews. 2014; 15(8):e344-53.

3. Govindaswami B, Jegatheesan P, Song D. Oxygen saturation screening for critical congenital heart disease. Neoreviews. 2012; 13(12):e724-31.
4 Guerchicoff M, Mrantz P, Infante J, Villa A, et al. Evaluación del impacto del diagnóstico precoz de las cardiopatías congénitas. Arch Argent Pediatr. 2004; 102(6):445-50.

5. Australian Institute of Health and Welfare. Heart, Stroke \& Vascular Diseases: Australian Facts 2004. AIHW No. CVD 27. Canberra: AIHW and National Heart Foundation of Australia; 2004. (Cardiovascular Disease Series No. 22).

6. Mahle WT, Clancy RR, Moss EM, Gerdes M, et al. Neurodevelopmental outcome and lifestyle assessment in schoolaged and adolescent children with hypoplastic left heart syndrome. Pediatrics. 2000; 105(5);1082-9.

7. Snookes SH, Gunn JK, Eldridge BJ, Donath SM, et al. A systematic review of motor and cognitive outcomes after early surgery for congenital heart disease. Pediatrics. 2010; 125(4):e818-27.

8. Gaynor JW, Stopp C, Wypij D, Andropoulos DB, et al. Neurodevelopmental outcomes after cardiac surgery in infancy. Pediatrics. 2015; 135(5):816-25.

9. Guyatt GH, Feeny DH, Patrick D. Measuring health-related quality of life. Ann Intern Med. 1993; 118(8): 622-9.

10. World Health Organization. WHOQOL: Measuring Quality of life. Geneva: WHOQOL Group: 1997.

11. Testa MA, Simonson DC. Assessment of quality-of-life outcomes. N Engl J Med. 1996; 334(13):835-40.

12. Mussatto K, Tweddell J. Quality of life following surgery for congenital cardiac malformations in neonates and infants. Cardiol Young. 2005; 15(Suppl 1):174-8.

13. Roizen M, Rodríguez S, Bauer G, Medin G, et al. Initial validation of the Argentinean Spanish version of the PedsQLTM 4.0 Generic Core Scales in children and adolescents with chronic diseases: acceptability and comprehensibility in low-income settings. Health Qual Life Outcomes. 2008; 6:59.

14. Grupo de trabajo sobre Calidad de Vida en la Infancia. Calidad de vida relacionada con la salud en pediatría. Instrumentos utilizados para su evaluación. Arch Argent Pediatr. 2014; 112(6):571-6.

15. Culbert EL, Ashburn DA, Cullen-Dean G, Joseph JA, et al. Quality of life of children after repair of transposition of the great arteries. Circulation. 2003; 108(7):857-62.

16. Møyen Laane K, Meberg A, Otterstad JE, Frøland G, et al. Quality of life in children with congenital heart defects. Acta Paediatr. 1997; 86(9):975-80.

17. Dunbar-Masterson C, Wypij D, Bellinger DC, Rappaport LA, et al. General health status of children with D-transposition of the great arteries after arterial switch operation. Circulation. 2001; 104(12 Suppl 1): I138-42.

18. Ekman-Joelsson BM, Berntsson L, Sunnergårdh J. Quality of life in children with pulmonary atresia and intact ventricular septum. Cardiol Young. 2004; 14(6):615-21.

19. Brosig CL, Mussatto KA, Kuhn EM, Tweddell JS. Psychosocial outcomes for preschool children and families after surgery for complex congenital heart disease. Pediatr Cardiol. 2007; 28(4):255-62.

20. Hovels-Gurich H, Konrad K, Wiesner M, Minkenberg R, et al. Long term behavioral outcome after neonatal arterial switch operation for transposition of the great arteries. Arch Dis Child. 2002; 87(6):506-10.

21. Lawoko S, Soares JJ. Quality of life among parents of children with congenital heart disease, parents of children with other diseases and parents of healthy children. Qual Life Res. 2003; 12(6):655-66.

22. Latal B, Helfricht S, Fischer JE, Bauersfeld U, Landolt MA. Psychological adjustment and quality of life in children and adolescents following open-heart surgery for congenital heart disease: a systematic review. BMC Pediatr. 2009; 9:6.

23. Roizen M, Figueroa C, Salvia L, Comité de Calidad de Vida 
y Salud. Calidad de vida relacionada con la salud en niños con enfermedades crónicas: comparación de la visión de los niños, sus padres y sus médicos. Arch Argent Pediatr. 2007; 105(4):305-13.

24. Garcia Guerra G, Robertson CM, Alton GY, Joffe AR, et al. Quality of life 4 years after complex heart surgery in infancy. J Thorac Cardiovasc Surg. 2013; 145(2):482-8.e2.
25. Sánchez C, Eymann A, De Cunto C, D'Agostino D. Quality of life in pediatric liver transplantation in a single-center in South America. Pediatr Transplant. 2010; 14(3):332-6.

26. Patel BJ, Lai L, Goldfield G, Sananes R, Longmuir PE. Psychosocial health and quality of life among children with cardiac diagnoses: agreement and discrepancies between parent and child reports. Cardiol Young. 2017; 27(4):713-21. 